\title{
Reduced precision underwrites ego dissolution and therapeutic outcomes under psychedelics
}

\author{
Devon Stoliker', Gary F. Egan ${ }^{1,2}$ Adeel Razi ${ }^{1,2,3,4}$ \\ ${ }^{1}$ Turner Institute for Brain and Mental Health, Monash University, Clayton, VIC, Australia \\ ${ }^{2}$ Monash Biomedical Imaging, Monash University, Clayton, VIC, Australia \\ ${ }^{3}$ Wellcome Centre for Human Neuroimaging, UCL, London, United Kingdom \\ ${ }^{4}$ CIFAR Azrieli Global Scholars Programs, CIFAR, Toronto, Canada
}

\begin{abstract}
Evidence suggests classic psychedelics reduce the precision of belief updating and enable access to a range of alternate hypotheses that underwrite how we make sense of the world. This process, in the higher cortices, has been postulated to explain the therapeutic efficacy of psychedelics for the treatment of internalising disorders. We argue reduced precision also underpins change to consciousness, known as 'ego dissolution', and that alterations to consciousness and attention under psychedelics have a common mechanism of reduced precision of Bayesian belief updating. Evidence connecting the role of serotonergic receptors to large-scale connectivity changes in the cortex suggests the precision of Bayesian belief updating may be a mechanism to modify and investigate consciousness and attention.
\end{abstract}

Keywords: precision, hierarchical predictive coding, psychedelics, ego dissolution, belief updating

\section{Introduction:}

Classic psychedelics, including psilocybin and lysergic diethylamide acid (LSD), at sufficient doses, can induce a range of subjective effects that include visual alterations (Studerus, Gamma, Kometer, \& Vollenweider, 2012), altered perception of time (Wittmann et al., 2007) 
and shifts in attention and perspective that reframe the relationship of self with the world (Swanson, 2018). This latter phenomenon, known as ego dissolution, is suggested to be the fundamental feature of classic psychedelic psychoactive effects (Stoliker, Egan, Friston, \& Razi, 2021). Ego dissolution, or the shift in sense of self and dissolution of boundary between the self and world (Lebedev et al., 2015; Millière, 2017), is a validated independent and measurable construct (Nour et al., 2016), with evidence suggesting that therapeutic outcomes are tied to its occurrence ${ }^{1}$ (R. L. Carhart-Harris \& Goodwin, 2017; Garcia-Romeu, Griffiths, \& Johnson, 2014; Majic, Schmidt, \& Gallinat, 2015; Roseman, Nutt, \& Carhart-Harris, 2018; Yaden \& Griffiths, 2020). Ego dissolution and all subjective effects from classic psychedelics originate from the binding kinetics of the 5HT2A receptor to psychedelic compounds (Preller et al., 2019; Quednow, Kometer, Geyer, \& Vollenweider, 2012) with other 5HT receptors are suggested to mediate psychedelic experiences (Vollenweider \& Preller, 2020). The 5HT2A is a serotonergic receptor involved in attention, especially in the prediction of the cause of sensory impressions and the consequences of self-initiated actions (Picard \& Friston, 2014).

The influence of psychedelics on serotonergic receptors are thought to underpin the veracity of beliefs by alterations to predictive processing (R. L. Carhart-Harris \& Friston, 2019). Predictive processing describes the interplay between ascending neuronal signals - in the form of ascending prediction errors — and descending predictions that embody internal world models (K. Friston, 2008; Rao \& Ballard, 1999). Synaptic communication between each layer of the cortical hierarchy and the layer immediately preceding it compares predictions and prediction errors to fulfil the core function of the brain: to reduce surprise (Clark, 2015; K. Friston, 2010; K. Friston \& Kiebel, 2009). Surprise (surprisal) is described mathematically by the Free Energy Principle (FEP), as minimising (variational) free energy (K. Friston \&

\footnotetext{
${ }^{1}$ Ego dissolution is thought to be synonymous with peak experiences and mystical experiences, which are also terms used to describe the main existential subjective effect of classic psychedelics and have been related to therapeutic outcomes.
} 
Kiebel, 2009; K. Friston, Kilner \& Harrison, 2006). Ascending signals which contradict predictions generate prediction errors which are 'surprising' and therefore garner attentional resources. Access to the appropriate level of the cortical processing hierarchy which hosts sufficient associative complexity is necessary to resolve the prediction error and thereby minimise surprise $^{2}$ (Millidge, 2021). This can occur through predictive processes that guide attention to update predictions (K. Friston, 2003) ${ }^{3}$ or by initiation of actions on the world to align sensory evidence with predictions (K. J. Friston, Daunizeau, \& Kiebel, 2009) ${ }^{4}$. Both strategies operate to minimise the difference (i.e., prediction error) between predictions and the sensory input.

The function of the cortical hierarchy to minimise surprise (or prediction errors) entails the maintenance of a generative model of the world which hosts beliefs about the causes and consequences of exogenous and endogenous sensations ${ }^{5}$ (Millidge, 2021; Parr \& Friston, 2018). A cornerstone of the generative aspect of the model is the ability to update beliefs ${ }^{6}$. Belief updating, known as Bayesian inference, represents a unifying principle in neural computation (Knill \& Pouget, 2004). According to Bayes' rule, the nervous system forms a model of the causal structure of the world by estimating the likely causes of sensory information. These estimations are derived by internal (generative) models of the causal structure of the world that are biased by prior beliefs. Once a causal explanation of prediction errors is produced, prior beliefs are able to update, and form new posterior beliefs (Bennett, 2015). Belief updating is (here suggested as) the process of moment-to-moment experience witnessed as consciousness and influenced by attention.

\footnotetext{
${ }^{2}$ Prediction errors are the information in each layer that is not predicted by descending predictions.

${ }^{3}$ Updating predictions can occur through immediate inference or updating the generative model of the world.

${ }^{4}$ Predictions are referred to as synonymous with beliefs.

${ }^{5}$ Both endogenous and exogenous signals are referred to as sensory signals/sensory impressions/sensory information.

${ }^{6}$ A key factor of belief updating involves beliefs about hidden variables of the world (i.e., variables which cannot be directly observed and rely on inference).
} 
Beyond the role of prediction of the nature of sensory signals, predictive processing also estimates a signal to noise ratio of prediction errors that modulate how prediction errors can be used to update predictions across the predictive hierarchy. This measure of certainty of sensory signals, known as precision, acts as a neural implementation of attention between layers of the cortical hierarchy-for example, in low levels of the hierarchy that precede conscious awareness (Millidge, 2021; Yon \& Frith, 2021). The generative model encodes the causes of sensory states, and the confidence of predictions, in predictive processes which are more or less independent of high level beliefs. For example, in the immediate (unconscious) inference about hidden states of the world through the construction of sensory impressions such as vision and audition ${ }^{7}$. Information gained from these lower-level sensory impressions may form a prediction error and entail an adjustment of higher-level beliefs. For example, the belief there is no vehicle approaching while crossing a road may be adjusted by receiving updated sensory information of the speedy approach of a vehicle, demonstrating that predictive processing between ascending sensation and descending cognition (i.e., predictions) coordinate to update beliefs. When the prediction of a particular level in the hierarchy requires an update (i.e., experiences contradiction), the prediction error is attended and granted access to higher levels where prediction errors are resolved. In the above case, high level cognitive beliefs are updated, and action may be taken to minimise the error in the prior belief to avoid being struck by a moving vehicle. This mechanism requires that sensory prediction errors have a pathway facilitated through automated (sub-personal) attention to a higher processing level where conscious cognitive processes are able to minimise an error using top down attentional resources. Predictive processing therefore suggests a means to

\footnotetext{
${ }^{7}$ This idea draws reference to Hermann Von Helmholtz's idea that perception is unconscious inference and the notion of uncertainty being computed in sub-personal processes beneath the level of subjective awareness (Yon \& Frith, 2021).
} 
examine and understand cognition — not an entirely new endeavour (Hohwy, 2020; Rorot, 2021). Attempts to bridge alternate methods of examining cognition, such as the analysis of large-scale resting state network connectivity, may aid the interpretation of predictive processes in consciousness and attention. Psychedelics present a unique opportunity to observe changes to large-scale connectivity in an altered state of consciousness that influences attention. We suggest precision — which corresponds to the modulation of prediction error signals underlying attention in predictive processing - may help unify multiscale connectivity changes and advance our understanding of consciousness and attention.

Precision - or the inverse of the variance of a signal — is explored in this work as a bridge that connects belief updating across predictive processing with large-scale brain connectivity. Precision is thought to play a key role in predictive processes that encode predictions of sensory stimuli by encoding the intrinsic uncertainty of predictions that select channels of attention and their salience (Adams, Stephan, Brown, Frith, \& Friston, 2013; Kanai, Komura, Shipp, \& Friston, 2015; Millidge, 2021). Precision is useful in a dynamic environment by allowing a generative model of any organism to estimate a level of uncertainty about beliefs and their causal construction. This affords the opportunity to grant flexibility that enables adaptation to changing contexts. Precision can be modelled as a probabilistic range of prior alternate hypotheses about the causes of sensory information. Very confident beliefs represent high precision of neuronal signalling and suggest a lower probability that alternate hypotheses will be cognitively explored through attention and selected. In the instance of crossing the road, a very precise belief that there is no oncoming traffic may preclude attentional mechanisms from initiating an action to visually scan the road before attempting to cross based on exogenous sensory evidence. However, more abstract beliefs related to endogenous sensory evidence and the generative model of the self and world are similarly 
able to undergo updating. For example, a prior belief about the honesty of a person may be updated by contradictory sensory information. Greater precision of beliefs at higher levels of the cortical processing hierarchy is indicative of an individual's confidence in the accuracy of their generative model of the world ${ }^{8}$. This confidence can modulate precision (and thus attentional resources) to the prediction errors at lower levels of the hierarchy (Yon \& Frith, 2021). Consequently, a high level of belief in the honesty of, for example, one's partner, may inhibit contrary sensory evidence from updating interpersonal or abstract (higher level) beliefs, which suggests a close association of precision to attention that underwrites the updating of conscious beliefs.

Despite precision being closely related to the self, it is significantly dependent on and modulated by neurotransmitter systems (Yon \& Frith, 2021). Upper levels of the cortical hierarchy involved in consciousness and attention are abundant in serotonergic 5HT2A receptors (Saulin, Savli, \& Lanzenberger, 2012) (R. L. Carhart-Harris \& Friston, 2019). The crucial role of serotonergic receptors in consciousness and attention is underscored by their function in the prediction of both the cause of sensory impressions and the consequences of self-initiated actions (Picard \& Friston, 2014). Accordingly, agonism of the serotonergic 5HT2A receptors by psychedelics is thought to reduce the precision of belief updating and reduce the 'confidence' (or certainty) in the selection of any one channel of attention (R. L. Carhart-Harris \& Friston, 2019). This has been postulated to afford a greater range of alternate hypotheses associated with the prior beliefs of a generative model (R. L. CarhartHarris \& Friston, 2019), see Fig. 2. The effect of psychedelics on precision is supported by electrophysiological evidence that suggests a 'flattening' of the free energy landscape (Bastos

\footnotetext{
${ }^{8}$ Precision is modulated by higher level cognition, such as cognitive biases, context and belief in the reliability of information. Exploration of these ideas is reviewed in (Yon \& Frith, 2021).
} 
et al., 2012), observations of increased entropy (Herzog et al., 2020) and theorised associations with increases in plasticity (R Carhart-Harris, 2021).

Ego dissolution underlies therapeutic benefits of psychedelics

The previously described mechanisms of precision and predictive processing, as they apply to therapeutic modes of belief updating under psychedelics, are outlined in the 2019 work of Carhart-Harris and Friston (R. L. Carhart-Harris \& Friston, 2019). They suggest the reduced precision of prior beliefs under psychedelics increases the range of hypotheses that can be entertained, and greater attentional resources allocated to alternate hypotheses as the product of reduced precision — with potentially therapeutic outcomes under proper guidance and supportive conditions. We extend this argument and suggest beliefs that undergird the self are updated in moment-to-moment experiences and that reduced precision of belief updating under psychedelics facilitates a change to consciousness (i.e., ego dissolution). Moreover, we suggest ego dissolution is a change in consciousness that reorients attention and enables therapeutic revisions of prior beliefs. This indicates the interplay between consciousness and attention are altered by classic psychedelics and underwritten by common changes to the precision of belief updating. The interplay between consciousness and attention is here generally hypothesised as attention producing consciousness and consciousness focusing attention. This formula follows a model of bottom up sensory predictive processing, and crucially precision mechanisms selecting channels of attention, that manifest in higher levels of the cortical hierarchy as conscious impressions. In turn, these conscious impressions influence how top down (higher) levels coordinate executive processes to focus attention. This theorisation of the interplay is consistent with earlier descriptions of consciousness that direct top down attention (Nani et al., 2019). Under this premise, we demonstrate that 
alignment between predictive processing and larger scale connectivity changes in the brain may be dependent on reduced precision of belief updating, and describe reduced precision of belief updating as the trigger to alterations in consciousness and attention.

The importance of the self in therapeutic outcomes, occurrences of ego dissolution and consciousness and attention inspire a bottom up and top down approach to understand the influence of precision of belief updating. The bottom up approach recognises consciousness as a constructive process of inference operating at the level of neural populations throughout the cortical hierarchy and that precision weighting influences the selection of attentional resources that integrate multimodal sensory connections (Millidge, 2021; Yon \& Frith, 2021). This type of integration has been suggested to form the basis of phenomenal consciousness (Gallagher, 2000; Millière, 2017; Seth, 2013) as a description of how reality appears to us (Block, 2007; Nani et al., 2019). We suggest change to precision of bottom up predictive processes may be important to psychedelic subjective effects, such as ego dissolution, and refer to large scale connectivity evidence that suggest the reduced filtering of the contents of consciousness under psychedelics is explained by filtration theory. Filtration theory describes the result of inhibition of filtering processes in the brain by the relaxation of constraints on perception, emotions, thought, and sense of self (Marshall, 2005; Osmond, 1957; Swanson, 2018). Change to bottom up filtration under the control of selection processes of attention may have significant implications for multimodal integration of the conscious sense of embodiment. Psychedelics have the potential to help investigate how bottom up changes to the precision of belief updating may be an important piece of the puzzle to understand changes of attention, consciousness and self. 
The top down approach acknowledges the role of precision in high-level cognition associated with large-scale resting state networks (Yon \& Frith, 2021). High levels of the cortical hierarchy integrate ascending multimodal signals with reference to the self and redirect attention based on goal directed salience (Menon, 2015). A focus on the upper most levels of the cortical hierarchy is pertinent to the exploration of psychedelics due to the abundant distribution of 5HT2A receptors at this level (R. L. Carhart-Harris \& Friston, 2019; Saulin et al., 2012). The receptor abundance is key as it may reduce the precision of predictions that have an inhibitory effect upon bottom up signals (R. L. Carhart-Harris \& Friston, 2019). Self-related aspects of phenomenal consciousness such as self-identity can be explored through functional magnetic resonance imaging (fMRI) studies that have associated connectivity of networks and regions to self-related functions and identified connectivity alteration in psychedelic-induced ego dissolution. Interpretation of this evidence with reference to the mechanism of precision suggests precision of belief updating may underwrite psychedelic-induced ego dissolution and by extension the therapeutic opportunities to redirect bottom up and top down attention in the ego dissolution state of consciousness. The exploration of precision under psychedelics focuses on the salience and default mode networks. The function of these networks in consciousness overlaps with the self (i.e., ego $^{9}$ ) and serves as a primary example of reduced precision, or confidence, that underpins the subjective experience of ego dissolution. Evidence of their 'disintegration' (reduced functional connectivity) under psychedelics may be explained by loss of precision leading to reduced belief updating of the model of self that produces occurrences of ego dissolution. However, firstly we turn our attention to the role of bottom up salience detection and discuss its potential role in bottom up precision of belief updating during experiences of ego dissolution.

\footnotetext{
${ }^{9}$ Narrative self, which is closely related to the Freudian concept of ego, is identified in relation to the default mode network (Buckner, Andrews-Hanna, \& Schacter, 2008; R. L. Carhart-Harris \& Friston, 2010; Lebedev et al., 2015).
} 


\section{Bottom up salience}

Phenomenal selfhood, also referred to as the minimal or embodied self, describes the basic, pre-reflective, sense of self as being rooted in sensory motor processes (Apps \& Tsakiris, 2014; Blanke \& Metzinger, 2009; Legrand \& Ruby, 2009; Limanowski \& Blankenburg, 2013). Embodiment connects and separates the self from the world. It relies upon the proper integration of multisensory signals from visual, somatosensory, interoceptive and vestibular feedback (Millière, 2017). The integration of multisensory signals through predictive processes describes the belief updating of sensory impressions and detection of salient features in the environment (Menon, 2015). Salience detection involves the fast, automatic, filtering of perceptual features that are infrequent or of biological importance, as detected along the ascending neural pathways of the predictive hierarchy (Knudsen, 2007). Change to the precision of bottom up predictive processes of belief updating may have a significant effect on multimodal integration that manifests in phenomenal consciousness and influences the experience of embodiment. Freer interactions identified lower in the cortical hierarchy under psychedelics support this idea (Robin Carhart-Harris, Kaelen, \& Nutt, 2014). Research applying dynamic causal modelling, which estimates effective connectivity between brain regions, suggest freer interactions may relate to reduced thalamic filtration of neural signals to the cortex under LSD. Recent research demonstrated increased effective connectivity from the thalamus to the posterior cingulate cortex under LSD (Preller et al., 2019) that may indicate the selection processes of attention that constrain the contents reaching consciousness become disinhibited by psychedelics (R. L. Carhart-Harris \& Friston, 2019; Preller et al., 2019). Functional MRI observations of freer interactions are also identified in the medial temporal lobe and are associated to heightened responses to sensory stimuli under psychedelics (R. Carhart-Harris et al., 2014; R. L. Carhart-Harris, 2018; R. L. Carhart-Harris et al., 2014; Kaelen 
et al., 2016). These findings correspond with the access of bottom up prediction errors to higher levels of the cortical processing hierarchy that suggest reduced constraints (i.e., filtration processes) upon consciousness may contribute to experiences of ego dissolution. These changes to the integration of sensory impressions may therefore be underwritten by reduced precision of belief updating.

\section{Top down salience}

The automatic detection of salience from incoming sensory impressions is complemented by a secondary, higher-level, top down system that similarly scans for salience. This brain system, known as the salience network, is context dependent and goal directs the focus of attention towards self-relevant, engaging or rewarding stimuli (Menon, 2015; Seeley et al., 2007). It also serves to facilitate reaction and access to attention and working memory (Sridharan, Levitin, \& Menon, 2008). Moreover, the salience network serves a key function coordinating attention between internal and external stimuli, which entail modes of consciousness (Sridharan et al., 2008). Reduced precision of top down salience predictions may alter the ordinary goal directed focus of attention and have effects on the subjective structure of conscious experience. Reduced salience network activity has been detected in association with ego dissolution in an fMRI study of psilocybin (Lebedev et al., 2015). Furthermore, the diminution of anti-correlated activity between salience and default mode networks under the control of the salience network has been observed under psilocybin (R. L. Carhart-Harris et al., 2012). These findings suggest salience network connectivity changes coincide with psychedelic peak effects and ego dissolution. The diminution of anti-correlation between large-scale brain networks may be one of the mechanisms of ego dissolution by reduced precision of the salience network and allocation of attention to alternate large-scale networks. Reduced precision in the salience allocation of attentional resources intuitively aligns with the dissolution of boundaries reported 
in ego dissolution. Additionally, the influence of freer bottom up ascending sensory impressions and reduced top down inhibition may compound to produce reduced constraints on consciousness. Reduced bottom up and top down precision underlying salience detection and the allocation of attention by the salience network are therefore hypothesised to jointly contribute to dissolving the sense of phenomenal self and the boundary between the internal and external world as reported in ego dissolution. We also argue that reduced precision of goal directed salience attention may contribute to a reprioritisation of salience that is important to extending attention to alternate high level psychosocial hypotheses and contribute to the utility of therapeutic applications of psychedelics.

\section{Top down narrative}

Salience detection describes a foundation of phenomenal consciousness through ascending predictive processes whilst higher order salience functions of the salience network suggest the concept of top down goal directed attention between internal and external modes of consciousness. The default mode network is also central to consciousness through its role in self-referential function and maintenance of passive awareness (Buckner et al., 2008; Raichle et al., 2001). Its expansion over the course of evolution (van Essen \& Dierker, 2007) suggests its core function to alert against predators during states of rest may have developed into sophisticated and uniquely human attributes, such as self-reflection (Qin et al., 2015) and a narrative sense of self. In Bayesian terms, the narrative self is the brain predicting its own contribution to sensory input by top down inferences about the causes (i.e., latent or hidden states of the world) of sensory information (Clark, 2013; K. Friston, 2010; Metzinger, 2003). The narrative sense of self is semantically closer to the familiar conscious experience of possessing beliefs. The default mode network has also been suggested as the candidate neural substrate of the Freudian conceptualisation of ego (Buckner et al., 2008; R. L. Carhart-Harris 
\& Friston, 2010; Lebedev et al., 2015). Disintegration (deactivation) of the default mode network is one of the most frequently cited neural correlates of psychedelic-induced ego dissolution (Ruban \& Kolodziej, 2018). Reduced functional connectivity of default mode network cardinal regions, such as the posterior cingulate cortex, are likely a key mechanism of disintegration and help to explain alterations of selfhood. The posterior cingulate cortex serves functions in self-reflection and its decreased oscillatory rhythms in the alpha range are observed under psilocybin (R. L. Carhart-Harris et al., 2016; Muthukumaraswamy et al., 2013). Notably, the range of oscillatory rhythms is thought to host beliefs in high level cortical areas (R. L. Carhart-Harris \& Friston, 2010). Cortical oscillations suggest a link between 5HT2A receptor agonism and observations of decreased functional connectivity of the default mode network under psychedelics. Translating the connectivity changes to psychological effects may be explained by reduced precision of belief updating. Such an interpretation carries intuitive appeal suggesting the basic function of region and network level connectivity underlies a sense of confidence that controls selection of attention among a range of alternatives, and that consistency in predictions or precision of predictions, underlies ordinary states of consciousness. The role of oscillations that bind micro and macro scales of connectivity is further demonstrated by evidence that oscillatory synchrony is a means by which conscious experience is unified (Singer, 1999). Synchronisation may be an expression of high precision. The influence of psychedelics to reduce precision and introduce greater cognitive flexibility aligns with the evidence that lagged synchronisation and agonist activity of the 5HT2A receptor underlies insightfulness: a facet of ego dissolution, with therapeutic potential. (Kometer, Pokorny, Seifritz, \& Volleinweider, 2015). This evidence may indicate a larger range of hypotheses that underlie belief updating form the foundation of self and world that are entertained under psychedelics, and that the overlap between ego dissolution and psychedelic therapeutic potential may share a common origin to reduce precision of belief updating. 
Revisiting the overlap between therapeutic outcomes and subjective effects and belief updating. Change to salience and the default mode network may affect the foundation of consciousness through altered multimodal integration, direction of attention - and its influence on modes of consciousness - and changes to a stable sense of self-identity. These are considered important facets of ego dissolution, that are underwritten by recurrent belief updating to maintain moment-to-moment conscious experience. Underlying large scale brain network connectivity changes, altered 5HT2A receptor activity affects patterns of synaptic gain and influence the selection of channels attended and the deployment of attentional resources (Kanai et al., 2015) (R. L. Carhart-Harris \& Friston, 2019). Changes to attention contribute to inferences underlying beliefs. Reduced precision affords attention to alternate hypotheses underlying selfrelated beliefs, and this association is critically linked by the necessity of self-dissolving subjective effects to produce therapeutic outcomes (Roseman et al., 2018; Yaden \& Griffiths, 2020). Although the association between psychedelic therapeutic effects and subjective effects remains debated, (Ogawa, Lee, Kay, \& Tank, 1990), the evidence put forward here suggests reduced precision underlies both effects. This idea is further supported by the alignment of mental health disorders, to which psychedelics show efficacy in therapy and their association with internalised self-beliefs. These include addiction, anxiety and depression (Robin CarhartHarris et al., 2021; R. L. Carhart-Harris et al., 2017; Griffiths, 2015; Johnson, Garcia-Romeu, Cosimano, \& Griffiths, 2014). These disorders, grouped as internalising conditions, involve maladaptive patterns of thought and behaviour underwritten by self-related beliefs.

Personal beliefs, similar to sub-personal beliefs, may be underwritten by a change to the precision of parameters that constitute a generative model of identity. Although 'belief updating' is more intuitively ascribed to beliefs one holds in association to oneself, one's high 
level generative model recurrently updates beliefs that constitute the nature of the self, from which self-held beliefs are hosted. The same predictive processes may then apply to both personal and sub-personal (automatic) forms of belief updating. Beliefs hosted by top down high-level cortical networks such as the salience network and default mode network are highly distributed with 5HT2A receptors (R. L. Carhart-Harris \& Friston, 2019). The reduced efficiency of predictive processes can explain decreases in the efficiency of attention to minimise prediction errors (Hohwy, 2012), and ego dissolution as the reduced precision of recurrent belief updating underlying attention that manifests the self. Precision may therefore be a fundamental mechanism of the ego, ego dissolution, and by association, therapeutic effects pertaining to attention underwriting self-related beliefs.

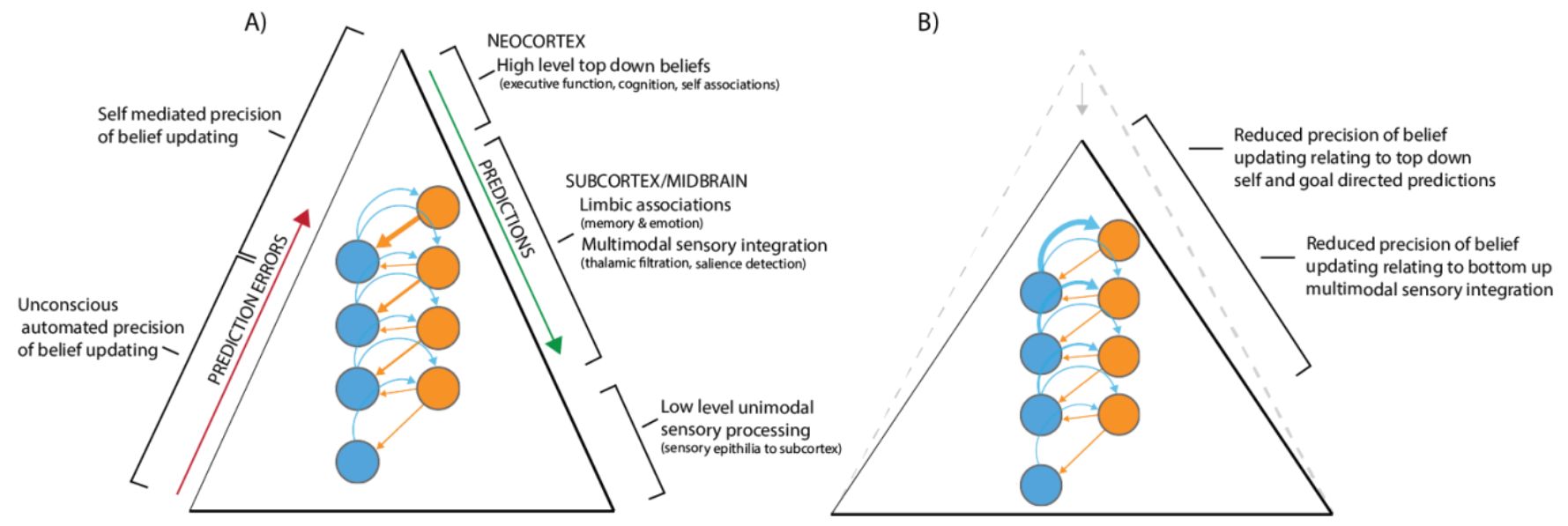

Figure 1. Simplified illustrative description of precision of belief updating along the cortical hierarchy. A) Precision is represented along scales of the hierarchy and depicts the filtration of sensory impressions to cognition. Predictions and prediction errors can be seen to interact between each layer of the hierarchy, with stronger high-level predictions in the upper levels of the hierarchy. B) Under psychedelics the precision of belief updating in high level predictions, involved in phenomenal consciousness and narrative self, is reduced. This occurs by belief updating opening alternate hypotheses that undergird neuronal populations 'confidence' in the selection, and salience, of attention that integrate phenomenal consciousness and narrative self-identity. Reduced precision is represented by the reduced weight of predictions and increased influence of prediction errors in higher levels of the hierarchy where 5HT2A receptors are abundant. The change to the precision of belief updating is hypothesised to result in ego dissolution. Elements of this model are inspired by CarhartHarris and Friston, 2019 (R. L. Carhart-Harris \& Friston, 2019).

\section{Desegregation and precision}


Figure 1 demonstrates the reduced 'top heaviness' and flattening of the cortical hierarchy by reduced precision of beliefs, as reflected by disintegration of connectivity in resting state networks and regional activity. The disintegration of higher levels of the cortical processing hierarchy is observed alongside a secondary outcome of psychedelic-induced connectivity dynamics, which may be relevant to the experience of the dissolution of boundaries and altered multimodal sensory integration. This second effect, coined desegregation, is shown to be positively correlated with ego dissolution (Tagliazucchi et al., 2016). Desegregation describes the abundant deviation of connectivity from functional pathways and is an effect cited in reference to the complexity of the psychedelic state (Barnett, Muthukumaraswamy, Carhart-Harris, \& Seth, 2019; Petri et al., 2014), see Figure 2. Desegregation in cortical communities may relate the richness of psychedelic phenomenological experience which differs from other altered self-dissolving states of consciousness (Millière, Carhart-Harris, Roseman, Trautwein, \& Berkovich-Ohana, 2018) that may relate to the complexity of a self generative model (Rorot, 2021).

Increased complexity in the psychedelic state may relate to the important relationship between complexity and accuracy. Efficient communication requires a balance between accuracy (precision) and complexity (information entertained) (Bishop, 2006). The increased complexity of cortical connections in the psychedelics state may influence the generative models access to alternative hypotheses (prior beliefs) and sensory information (freer low level interactions). Consequently, precision or confidence in the accuracy of any one prior belief may be reduced and stretch the imagination or range of alternative hypotheses within 
the generative model. This may dispose attention to unfamiliar hypotheses (and selection of connectivity pathways ${ }^{10}$ ) that underly the model of self, and social exchanges with the world.
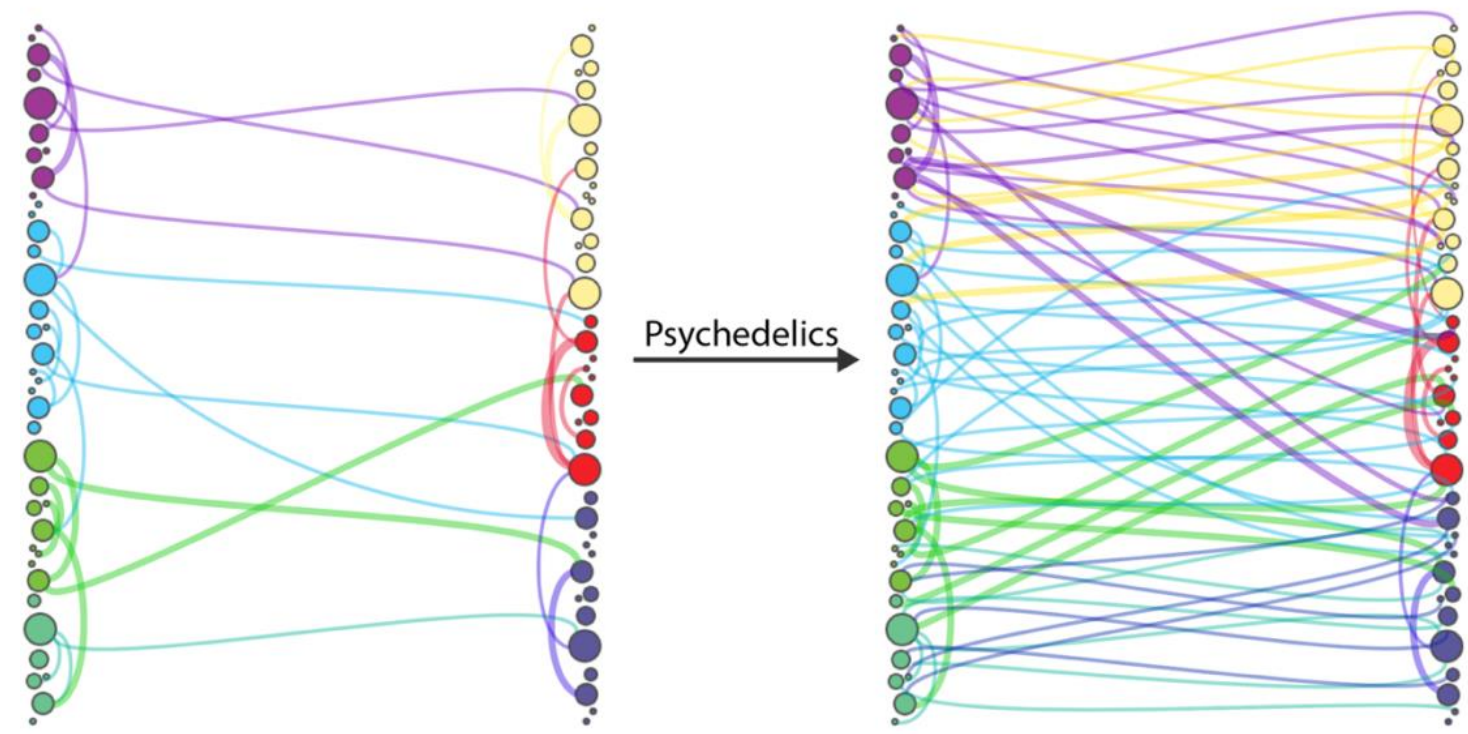

Figure 2. Illustration of desegregated connectivity under psilocybin, inspired by Petri et. al., 2014. Change between cortical communities' diversity of connectivity under psilocybin suggests a foundation of subjective effects (and facet of ego dissolution) that may be underwritten by reduced precision (i.e., confidence) of belief updating enabling connectivity to select from a wider range of hypotheses.

\section{Conclusions}

Brain connectivity changes observed in psychedelic imaging research may be explained by the reduced precision of neuronal signalling in top down and bottom up hierarchical predictive processes. Under psychedelics, changes to precision are suggested to extend attentional resources to a broader range of hypotheses that operate to minimise prediction errors. Reduced efficiency of error minimisation induced by psychedelics can offer the counter intuitive effect of breaking underlying maladaptive patterns of self-belief and offer attention to alternate hypotheses underlying construction of the world. In the light of evidence

\footnotetext{
${ }^{10}$ If we imagined the distribution of variance as a sort of compass, we might hypothesise some association between reduced precision selecting alternative hypotheses and the desegregation of large-scale connectivity pathways.
} 
that psychedelics induce ego dissolution and its association to therapeutic outcomes, we argue that reduced precision alters belief updating which undergirds the phenomenal and narrative self, that is experienced as ego dissolution. Psychedelic therapeutic effects may rely on the subjective change to consciousness, through a common change to attention processes underwritten by precision. Phenomenal consciousness and the sense of self are suggested as key facets of ego dissolution, that are affected by the reduced confidence in reiterative belief updating, under the action of psychedelics.

Precision is suggested to be ubiquitous in the nervous system. Precision bridges predictive processes across unconscious and conscious levels by acting as a measure of confidence. A natural extension to this is that precision of belief updating will then apply to numerous psychedelic effects such as visual hallucinations. Beyond psychedelics, precision may also apply to wellbeing, psychopathology and by extension, basic facets of cortical function and perception. Precision may be one step in a complex sequence of interacting predictive process mechanisms. The necessity to comprehensively synthesise mechanisms of precision associated with the dynamics of consciousness and attention are acknowledged, as well as the limitation that precision alone may not provide an exhaustive account of predictive processing or connectivity mechanisms. Other facets of predictive processing, such as reference to the complexity of the generative model, interaction of precision across the hierarchy and with the environment are equally important ${ }^{11}$. However, precision does provide an intuitive way to interpret the translation of neural processes to consciousness.

Conceptualising precision as a key mechanism holds utility as an intuitive interpretation that explains the necessity of living organisms to recurrently update their beliefs to remain adaptive to an ever-changing environment, and more fundamentally, to remain conscious of

\footnotetext{
${ }^{11}$ Complexity is suggested as an important feature alongside precision. Interested readers are directed to (Rorot, 2021). For review of precision and the Bayesian brain see (Yon \& Frith, 2021).
} 
their self and surroundings. This suggests the self is a constructive process of ongoing updating and that a useful way to understand predictive processing — both conscious and unconscious - is in terms of 'beliefs'. Beliefs can be determined by precision, which represents a form of confidence, that at one end is psychological in nature, and at the other occurs in automatic sub-personal neuronal processes. Furthering our understanding of the relationship between the conscious and unconscious, and its relationship to attention, suggests a key utility of psychedelics for research in the cognitive sciences to experimentally probe predictive process theories of consciousness and attention ${ }^{12}$ and test their theoretical robustness.

\section{Conflict of interest}

The authors declare no conflicts of interest.

Author Contributions:

Devon Stoliker: Conceptualization \& writing.

Gary F. Egan: Writing—review \& editing.

Adeel Razi: Writing—review \& editing.

\section{Acknowledgements:}

AR is funded by the Australian Research Council (Refs: DE170100128 I\& DP200100757) and Australian National Health and Medical Research Council Investigator Grant (Ref: 1194910). AR is a CIFAR Azrieli Global Scholar in the Brain, Mind $\backslash \&$ Consciousness

12 These theories are not explored in this review. Interested readers are directed to (Hohwy, 2020) and (Rorot, 2021). 
Program. AR and NS are affiliated with The Wellcome Centre for Human Neuroimaging

supported by core funding from Wellcome [203147/Z/16/Z].

\section{References:}

Adams, R., Stephan, K., Brown, H., Frith, C., \& Friston, K. (2013). The Computational Anatomy of Psychosis. Frontiers in Psychiatry, 4(47). doi:10.3389/fpsyt.2013.00047

Apps, M. A., \& Tsakiris, M. (2014). The free-energy self: a predictive coding account of selfrecognition. Neurosci Biobehav Rev, 41, 85-97. doi:10.1016/j.neubiorev.2013.01.029

Barnett, L., Muthukumaraswamy, S. D., Carhart-Harris, R. L., \& Seth, A. K. (2019). Decreased Directed Functional Connectivity in the Psychedelic State. bioRxiv, 703660. doi:10.1101/703660

Bastos, A. M., Usrey, W. M., Adams, R. A., Mangun, G. R., Fries, P., \& Friston, K. J. (2012). Canonical microcircuits for predictive coding. Neuron, 76(4), 695-711. doi:10.1016/j.neuron.2012.10.038

Bennett, D. (2015). The Neural Mechanisms of Bayesian Belief Updating. The Journal of Neuroscience, 35(50), 16300. doi:10.1523/JNEUROSCI.3742-15.2015

Bishop, C. M. (2006). Pattern Recognition and Machine Learning (Information Science and Statistics): Springer-Verlag.

Blanke, O., \& Metzinger, T. (2009). Full-body illusions and minimal phenomenal selfhood. Trends Cogn Sci, 13(1), 7-13. doi:10.1016/j.tics.2008.10.003

Block, N. (2007). Consciousness, accessibility, and the mesh between psychology and neuroscience. Behav Brain Sci, 30(5-6), 481-499; discussion 499-548. doi:10.1017/s0140525x07002786

Buckner, R. L., Andrews-Hanna, J. R., \& Schacter, D. L. (2008). The brain's default network: anatomy, function, and relevance to disease. Ann N Y Acad Sci, 1124, 1-38. doi:10.1196/annals.1440.011

Carhart-Harris, R. (2021). Psychedelics, entropy and plasticity. Retrieved from https://psychedelicstoday.com/2021/05/25/pt245-robin-carhart-harris-psychedelicsentropy-and-plasticity/

Carhart-Harris, R., Giribaldi, B., Watts, R., Baker-Jones, M., Murphy-Beiner, A., Murphy, R., . . Nutt, D. J. (2021). Trial of Psilocybin versus Escitalopram for Depression. New England Journal of Medicine, 384(15), 1402-1411. doi:10.1056/NEJMoa2032994

Carhart-Harris, R., Kaelen, M., \& Nutt, D. (2014). How do hallucinogens work on the brain? Psychologist, 27(9), 662-665. Retrieved from <Go to ISI>://WOS:000341172000027

Carhart-Harris, R. L. (2018). The entropic brain - revisited. Neuropharmacology, 142, 167 178. doi:10.1016/j.neuropharm.2018.03.010

Carhart-Harris, R. L., \& Friston, K. J. (2010). The default-mode, ego-functions and freeenergy: a neurobiological account of Freudian ideas. Brain, 133(Pt 4), 1265-1283. doi:10.1093/brain/awq010

Carhart-Harris, R. L., \& Friston, K. J. (2019). REBUS and the Anarchic Brain: Toward a Unified Model of the Brain Action of Psychedelics. Pharmacological Reviews, 71(3), 316-344. doi:10.1124/pr.118.017160

Carhart-Harris, R. L., \& Goodwin, G. M. (2017). The Therapeutic Potential of Psychedelic Drugs: Past, Present, and Future. Neuropsychopharmacology : official publication of 
the American College of Neuropsychopharmacology, 42(11), 2105-2113. doi:10.1038/npp.2017.84

Carhart-Harris, R. L., Leech, R., Erritzoe, D., Williams, T. M., Stone, J. M., Evans, J., . . Nutt, D. J. (2012). Functional Connectivity Measures After Psilocybin Inform a Novel Hypothesis of Early Psychosis. Schizophrenia Bulletin, 39(6), 1343-1351. doi:10.1093/schbul/sbs117

Carhart-Harris, R. L., Leech, R., Hellyer, P. J., Shanahan, M., Feilding, A., Tagliazucchi, E., . . Nutt, D. (2014). The entropic brain: a theory of conscious states informed by neuroimaging research with psychedelic drugs. Frontiers in Human Neuroscience, 8. doi:10.3389/fnhum.2014.00020

Carhart-Harris, R. L., Muthukumaraswamy, S., Roseman, L., Kaelen, M., Droog, W., Murphy, K., . . Nutt, D. J. (2016). Neural correlates of the LSD experience revealed by multimodal neuroimaging. Proceedings of the National Academy of Sciences of the United States of America, 113(17), 4853-4858. Retrieved from https://www-jstororg.ezproxy.lib.monash.edu.au/stable/26469438

http://spiral.imperial.ac.uk/bitstream/10044/1/50366/5/PNAS-2016-Carhart-Harris-48538.pdf

Carhart-Harris, R. L., Roseman, L., Bolstridge, M., Demetriou, L., Pannekoek, J. N., Wall, M. B., . . . Nutt, D. J. (2017). Psilocybin for treatment-resistant depression: fMRImeasured brain mechanisms. Scientific Reports, 7(1), 13187. doi:10.1038/s41598$017-13282-7$

Clark, A. (2013). Whatever next? Predictive brains, situated agents, and the future of cognitive science. Behav Brain Sci, 36(3), 181-204. doi:10.1017/s0140525x12000477

Clark, A. (2015). Surfing uncertainty: Prediction, action, and the embodied mind.: Oxford University Press.

Friston, K. (2003). Learning and inference in the brain. Neural Netw, 16(9), 1325-1352. doi:10.1016/j.neunet.2003.06.005

Friston, K. (2008). Hierarchical Models in the Brain. PLOS Computational Biology, 4(11), e1000211. doi:10.1371/journal.pcbi.1000211

Friston, K. (2010). The free-energy principle: a unified brain theory? Nature Reviews Neuroscience, 11, 127. doi:10.1038/nrn2787

https://www.nature.com/articles/nrn2787\#supplementary-information

Friston, K., \& Kiebel, S. (2009). Predictive Coding under the Free-Energy Principle. Philosophical Transactions: Biological Sciences, 364(1521), 1211-1221. Retrieved from http://www.jstor.org.ezproxy.lib.monash.edu.au/stable/40485889

Friston, K., Kilner, J., \& Harrison, L. (2006). A free energy principle for the brain. J Physiol Paris, 100(1-3), 70-87. doi:10.1016/j.jphysparis.2006.10.001

Friston, K. J., Daunizeau, J., \& Kiebel, S. J. (2009). Reinforcement Learning or Active Inference? Plos One, 4(7), e6421. doi:10.1371/journal.pone.0006421

Gallagher, S. (2000). Gallagher, S. 2000. Philosophical Conceptions of the Self: Implications for Cognitive Science. Trends in Cognitive Sciences, 4, 14-21. doi:10.1016/S13646613(99)01417-5

Garcia-Romeu, A., Griffiths, R. R., \& Johnson, M. W. (2014). Psilocybin-occasioned mystical experiences in the treatment of tobacco addiction. Current drug abuse reviews, 7(3), 157-164. doi:10.2174/1874473708666150107121331

Griffiths, R. (2015). A Single Dose of Psilocybin Produces Substantial and Enduring Decreases in Anxiety and Depression in Patients with a Life-Threatening Cancer Diagnosis: A Randomized Double-blind Trial. Neuropsychopharmacology, 40, S90S91. Retrieved from <Go to ISI>://WOS:000366597700181 
Herzog, R., Mediano, P. A. M., Rosas, F. E., Carhart-Harris, R., Perl, Y. S., Tagliazucchi, E., $\&$ Cofre, R. (2020). A mechanistic model of the neural entropy increase elicited by psychedelic drugs. Scientific Reports, 10(1), 17725. doi:10.1038/s41598-020-74060-6

Hohwy, J. (2012). Attention and Conscious Perception in the Hypothesis Testing Brain. Frontiers in Psychology, 3(96). doi:10.3389/fpsyg.2012.00096

Hohwy, J. (2020). New directions in predictive processing. Mind \& Language, 35(2), 209223. doi:https://doi.org/10.1111/mila.12281

Johnson, M. W., Garcia-Romeu, A., Cosimano, M. P., \& Griffiths, R. R. (2014). Pilot study of the 5-HT2AR agonist psilocybin in the treatment of tobacco addiction. Journal of psychopharmacology (Oxford, England), 28(11), 983-992. doi:10.1177/0269881114548296

Kaelen, M., Roseman, L., Kahan, J., Santos-Ribeiro, A., Orban, C., Lorenz, R., . . CarhartHarris, R. (2016). LSD modulates music-induced imagery via changes in parahippocampal connectivity. European Neuropsychopharmacology, 26(7), 10991109. doi:https://doi.org/10.1016/j.euroneuro.2016.03.018

Kanai, R., Komura, Y., Shipp, S., \& Friston, K. (2015). Cerebral hierarchies: predictive processing, precision and the pulvinar. Philosophical Transactions of the Royal Society B: Biological Sciences, 370(1668), 20140169. doi:10.1098/rstb.2014.0169

Knill, D. C., \& Pouget, A. (2004). The Bayesian brain: the role of uncertainty in neural coding and computation. Trends Neurosci, 27(12), 712-719. doi:10.1016/j.tins.2004.10.007

Knudsen, E. I. (2007). Fundamental Components of Attention. Annu Rev Neurosci, 30(1), 57 78. doi:10.1146/annurev.neuro.30.051606.094256

Kometer, M., Pokorny, T., Seifritz, E., \& Volleinweider, F. X. (2015). Psilocybin-induced spiritual experiences and insightfulness are associated with synchronization of neuronal oscillations. Psychopharmacology, 232(19), 3663-3676. doi:10.1007/s00213-015-4026-7

Lebedev, A. V., Lövdén, M., Rosenthal, G., Feilding, A., Nutt, D. J., \& Carhart-Harris, R. L. (2015). Finding the self by losing the self: Neural correlates of ego-dissolution under psilocybin. Human Brain Mapping, 36(8), 3137-3153. doi:10.1002/hbm.22833

Legrand, D., \& Ruby, P. (2009). What is self-specific? Theoretical investigation and critical review of neuroimaging results [American Psychological Association doi:10.1037/a0014172]. Retrieved

Limanowski, J., \& Blankenburg, F. (2013). Minimal self-models and the free energy principle. Frontiers in Human Neuroscience, 7, 547-547. doi:10.3389/fnhum.2013.00547

Majic, T., Schmidt, T. T., \& Gallinat, J. (2015). Peak experiences and the afterglow phenomenon: when and how do therapeutic effects of hallucinogens depend on psychedelic experiences? J Psychopharmacol, 29(3), 241-253. doi:10.1177/0269881114568040

Marshall, P. (2005). Mind Beyond the Brain: Reducing Valves and Metaphysics. Oxford: Oxford University Press.

Menon, V. (2015). Salience Network (Vol. 2): Elsevier.

Metzinger, T. (2003). Being no one: The self-model theory of subjectivity. Cambridge, MA, US: MIT Press.

Millidge, B. S., A.; Buckley, C.L. (2021). Predictive Coding: a Theoretical and Experimental Review.

Millière, R. (2017). Looking for the Self: Phenomenology, Neurophysiology and Philosophical Significance of Drug-induced Ego Dissolution. Frontiers in Human Neuroscience, 11(245). doi:10.3389/fnhum.2017.00245 
Millière, R., Carhart-Harris, R. L., Roseman, L., Trautwein, F.-M., \& Berkovich-Ohana, A. (2018). Psychedelics, Meditation, and Self-Consciousness. Frontiers in Psychology, 9(1475). doi:10.3389/fpsyg.2018.01475

Muthukumaraswamy, S. D., Carhart-Harris, R. L., Moran, R. J., Brookes, M. J., Williams, T. M., Errtizoe, D., . . . Nutt, D. J. (2013). Broadband Cortical Desynchronization Underlies the Human Psychedelic State. The Journal of Neuroscience, 33(38), 1517115183. doi:10.1523/jneurosci.2063-13.2013

Nani, A., Manuello, J., Mancuso, L., Liloia, D., Costa, T., \& Cauda, F. (2019). The Neural Correlates of Consciousness and Attention: Two Sister Processes of the Brain.

Frontiers in Neuroscience, 13(1169). doi:10.3389/fnins.2019.01169

Ogawa, S., Lee, T. M., Kay, A. R., \& Tank, D. W. (1990). Brain magnetic resonance imaging with contrast dependent on blood oxygenation. Proceedings of the National Academy of Sciences of the United States of America, 87(24), 9868-9872. doi:10.1073/pnas.87.24.9868

Osmond, H. (1957). A review of the clinical effects of psychotomimetic agents. Ann N Y Acad Sci, 66(3), 418-434. doi:10.1111/j.1749-6632.1957.tb40738.x

Parr, T., \& Friston, K. J. (2018). The Anatomy of Inference: Generative Models and Brain Structure. Frontiers in Computational Neuroscience, 12(90). doi:10.3389/fncom.2018.00090

Petri, G., Expert, P., Turkheimer, F., Carhart-Harris, R., Nutt, D., Hellyer, P. J., \& Vaccarino, F. (2014). Homological scaffolds of brain functional networks. Journal of The Royal Society Interface, 11(101), 20140873. doi:10.1098/rsif.2014.0873

Picard, F., \& Friston, K. (2014). Predictions, perception, and a sense of self. Neurology, 83. doi:10.1212/WNL.0000000000000798

Preller, K. H., Razi, A., Zeidman, P., Stämpfli, P., Friston, K. J., \& Vollenweider, F. X. (2019). Effective connectivity changes in LSD-induced altered states of consciousness in humans. Proceedings of the National Academy of Sciences, 116(7), 2743-2748. doi:10.1073/pnas.1815129116

Qin, P., Wu, X., Huang, Z., Duncan, N. W., Tang, W., Wolff, A., . . Northoff, G. (2015). How are different neural networks related to consciousness? Ann Neurol, 78(4), 594605. doi:10.1002/ana.24479

Quednow, B. B., Kometer, M., Geyer, M. A., \& Vollenweider, F. X. (2012). PsilocybinInduced Deficits in Automatic and Controlled Inhibition are Attenuated by Ketanserin in Healthy Human Volunteers. Neuropsychopharmacology, 37(3), 630-640. doi:10.1038/npp.2011.228

Raichle, M. E., MacLeod, A. M., Snyder, A. Z., Powers, W. J., Gusnard, D. A., \& Shulman, G. L. (2001). A default mode of brain function. Proc Natl Acad Sci U S A, 98(2), 676682. doi:10.1073/pnas.98.2.676

Rao, R. P. N., \& Ballard, D. H. (1999). Predictive coding in the visual cortex: a functional interpretation of some extra-classical receptive-field effects. Nature Neuroscience, 2(1), 79-87. doi:10.1038/4580

Rorot, W. (2021). Bayesian theories of consciousness: a review in search for a minimal unifying model. Neuroscience of Consciousness, 2021(2). doi:10.1093/nc/niab038

Roseman, L., Nutt, D. J., \& Carhart-Harris, R. L. (2018). Quality of Acute Psychedelic Experience Predicts Therapeutic Efficacy of Psilocybin for Treatment-Resistant Depression. Frontiers in Pharmacology, 8, 974. doi:10.3389/fphar.2017.00974

Ruban, A., \& Kolodziej, A. A. (2018). Changes in default-mode network activity and functional connectivity as an indicator of psychedelic-assisted psychotherapy effectiveness. Neuropsychiatria I Neuropsychologia, 13(3), 91-97. doi:10.5114/nan.2018.81249 
Saulin, A., Savli, M., \& Lanzenberger, R. (2012). Serotonin and molecular neuroimaging in humans using PET. Amino Acids, 42(6), 2039-2057. doi:10.1007/s00726-011-1078-9

Seeley, W. W., Menon, V., Schatzberg, A. F., Keller, J., Glover, G. H., Kenna, H., . . . Greicius, M. D. (2007). Dissociable intrinsic connectivity networks for salience processing and executive control. The Journal of Neuroscience, 27(9), 2349-2356. doi:10.1523/jneurosci.5587-06.2007

Seth, A. K. (2013). Interoceptive inference, emotion, and the embodied self. Trends Cogn Sci, 17(11), 565-573. doi:10.1016/j.tics.2013.09.007

Singer, W. (1999). Neuronal Synchrony: A Versatile Code for the Definition of Relations? Neuron, 24(1), 49-65. doi:10.1016/S0896-6273(00)80821-1

Sridharan, D., Levitin, D. J., \& Menon, V. (2008). A critical role for the right fronto-insular cortex in switching between central-executive and default-mode networks. Proceedings of the National Academy of Sciences, 105(34), 12569. doi:10.1073/pnas.0800005105

Stoliker, D., Egan, G., Friston, K., \& Razi, A. (2021). Neural Mechanisms and Psychology of Psychedelic Ego Dissolution. In: PsyArXiv.

Studerus, E., Gamma, A., Kometer, M., \& Vollenweider, F. X. (2012). Prediction of Psilocybin Response in Healthy Volunteers. Plos One, 7(2). doi:10.1371/journal.pone.0030800

Swanson, L. R. (2018). Unifying Theories of Psychedelic Drug Effects. Frontiers in Pharmacology, 9(172). doi:10.3389/fphar.2018.00172

Tagliazucchi, E., Roseman, L., Kaelen, M., Orban, C., Muthukumaraswamy, S. D., Murphy, K., . . . Carhart-Harris, R. (2016). Increased Global Functional Connectivity Correlates with LSD-Induced Ego Dissolution. Current biology: CB, 26(8), 10431050. doi:10.1016/j.cub.2016.02.010

Van Essen, D. C., \& Dierker, D. L. (2007). Surface-based and probabilistic atlases of primate cerebral cortex. Neuron, 56(2), 209-225. doi:10.1016/j.neuron.2007.10.015

Vollenweider, F. X., \& Preller, K. H. (2020). Psychedelic drugs: neurobiology and potential for treatment of psychiatric disorders. Nature Reviews Neuroscience, 21(11), 611-624. doi:10.1038/s41583-020-0367-2

Wittmann, M., Carter, O., Hasler, F., Cahn, B. R., Grimberg, U., Spring, P., . . . Vollenweider, F. X. (2007). Effects of psilocybin on time perception and temporal control of behaviour in humans. Journal of Psychopharmacology, 21(1), 50-64. doi:10.1177/0269881106065859

Yaden, D. B., \& Griffiths, R. R. (2020). The Subjective Effects of Psychedelics Are Necessary for Their Enduring Therapeutic Effects. ACS Pharmacology \& Translational Science. doi:10.1021/acsptsci.0c00194

Yon, D., \& Frith, C. D. (2021). Precision and the Bayesian brain. Current Biology, 31(17), R1026-R1032. doi:10.1016/j.cub.2021.07.044 